\title{
Joint Channel Allocation, Interface Assignment and MAC Design for Multi-Channel Wireless Mesh Networks
}

\author{
A. Hamed Mohsenian Rad and Vincent W.S. Wong \\ Department of Electrical and Computer Engineering \\ The University of British Columbia, Vancouver, Canada \\ e-mail: \{hamed, vincentw\}@ece.ubc.ca
}

\begin{abstract}
In a wireless mesh network (WMN) with a number of stationary wireless routers, the aggregate capacity can be increased when each router is equipped with multiple network interface cards (NICs) and each NIC within a router is assigned to a distinct orthogonal frequency channel. In this paper, given the logical topology of the network, we formulate the joint channel allocation, interface assignment, and media access control (MAC) problem as a cross-layer non-linear mixed-integer network utility maximization problem. An optimal joint design, based on exact binary linearization techniques, is proposed which leads to a global maximum. A near-optimal joint design, based on approximate dual decomposition techniques, is also proposed which is of more interest in terms of practical deployment. Performance evaluation is given through a number of numerical examples in terms of network utility maximization and aggregate network throughput.
\end{abstract}

\section{INTRODUCTION}

Wireless Mesh Networks (WMNs) are becoming a popular alternative in extending the coverage of the Wireless Local Area Networks (WLANs) [1]. Mesh networks consist of mobile wireless clients and stationary wireless mesh routers. Mesh routers are connected to one another in a multi-hop manner to form a large scale wireless backbone. Some of the routers also act as gateways to the Internet via high-speed wired links. The performance of the IEEE $802.11 \mathrm{a} / \mathrm{b} / \mathrm{g}$-based WMNs can be increased via the use of multiple channels [2]. In this scenario, each router is equipped with multiple network interface cards (NICs). Each NIC is assigned to a distinct frequency channel. Two neighboring routers are able to communicate (and establish a logical link) with each other as long as one of their NICs uses the same channel. A sample multi-channel wireless mesh network (MC-WMN) is illustrated in Fig. 1.

Within the IEEE $802.11 \mathrm{a} / \mathrm{b} / \mathrm{g}$ frequency bands, the number of available channels is limited. The $802.11 \mathrm{~b} / \mathrm{g}$ bands and the 802.11a band provide 3 and 12 non-overlapping frequency channels, respectively. This implies that some logical links may operate over the same channel. The number of NICs is also limited. In the experimental MC-WMN test-beds in [2] and [3], each router is equipped with two NICs. A small number of NICs implies that some logical links in a router may need to share an NIC to transmit and receive data packets. Two nearby links that operate over the same channel or share

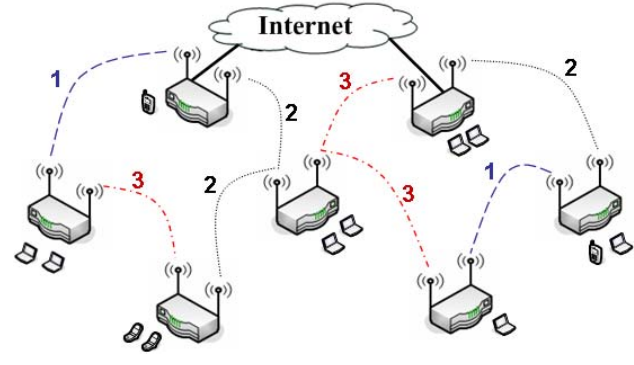

Fig. 1. A multi-channel wireless mesh network with seven routers, eight links, two network interface cards per router, and three frequency channels.

the same NIC cannot be active (i.e., transmit or receive data) simultaneously. It reduces the links' effective capacities. Given the logical topology of an MC-WMN, two important issues should be addressed: channel allocation and interface assignment. The former determines over which frequency channel each logical link should operate and the latter determines which logical links should share an NIC on each router.

The channel allocation and interface assignment schemes can be classified as static or dynamic algorithms [4]. The static algorithms assign channels and interfaces permanently [5]-[7]. In dynamic algorithms, the assigned channels and interfaces are updated either in a short-term basis (e.g., packet by packet [8]-[11]) or a long-term basis (e.g., every several minutes or hours [3], [12]). The dynamic algorithms allow the MC-WMN to adapt to the changing traffic patterns. Unlike the longterm basis algorithms, the short-term basis schemes require a fast coordination mechanism to ensure that the sending and receiving routers use the same channel, which can significantly increase the signalling overhead [3].

Various joint design schemes for MC-WMNs have been proposed recently. Some of the recent work include joint channel allocation and routing [13]-[15], joint routing and interface assignment [4], joint topology control and interface assignment [16], joint routing and media access control [17], joint topology control and routing [18], joint power control and channel allocation [19], and joint channel allocation and congestion control [20].

In this paper, we formulate a joint channel allocation, interface assignment, and media access control (MAC) problem as a cross-layer network utility maximization with constraints 
that arise from the interference among the neighboring transmissions. The contributions of our work are as follows:

- We model the channel allocation and interface assignment problems by introducing channel allocation and interface assignment binary vectors.

- We model the feasible region for the link-layer flow rates as a function of the allocated channels and assigned interfaces.

- We present a formulation for joint channel allocation, interface assignment, and MAC problem as a non-linear mixed-integer network utility maximization. It takes into account the number of NICs at each wireless mesh router, the number of orthogonal frequency channels, and the interference constraints.

- We solve the network utility maximization problem via both exact and approximate design schemes. The exact design results in an optimal static algorithm while the approximate design results in a near-optimal long-term basis dynamic algorithm.

The rest of this paper is organized as follows. The problem formulation is described in Section II. The first design scheme (using exact binary linearization) is presented in Section III. The second design scheme (using approximate dual decomposition) is described in Section IV. Performance evaluation and numerical examples are given in Section V. Conclusions and future work are given in Section VI.

\section{PROBLEM Formulation}

Consider an MC-WMN and let $N$ denote the set of stationary wireless mesh routers ${ }^{1}$. Each router is equipped with $I$ NICs. There are $C$ orthogonal frequency channels available. We assume that the network's logical topology has been predetermined. Let $L$ denote the set of all unidirectional logical links. The logical link from node $m$ to node $n$ is denoted by $(m, n) \in L$. We assume the connectivity to be symmetric. That is, link $(m, n) \in L$ if and only if $(n, m) \in L$.

In this section, we describe the mathematical model to formulate a joint channel allocation, interface assignment, and MAC problem.

\section{A. Channel Allocation Model}

For any two nodes $m, n \in N$ such that there exists a logical link $(m, n) \in L$, we define a $C \times 1$ channel allocation vector $\overline{\mathbf{x}}_{m n}$. If node $m$ communicates with node $n$ over the $i^{\text {th }}$ frequency channel, then the $i^{t h}$ element in $\overline{\mathbf{x}}_{m n}$ is equal to 1 ; otherwise, it is equal to zero. As an example, if $C=5$ and node $m$ is assigned to communicate with node $n$ over the second channel, then we have, $\overline{\mathbf{x}}_{m n}=\left[\begin{array}{lllll}0 & 1 & 0 & 0 & 0\end{array}\right]^{T}$. In this paper, we assume that there will never be more than one connection between any two nodes.

To establish the logical link $(m, n) \in L$, routers $m$ and $n$ should allocate a common frequency channel to communicate

\footnotetext{
${ }^{1}$ For the rest of this paper, the terms wireless mesh routers and nodes will be used interchangeably.
}

with each other. This requires that,

$$
\overline{\mathbf{x}}_{m n}=\overline{\mathbf{x}}_{n m}, \quad \forall m, n \in N, \quad(m, n) \in L
$$

and

$$
\mathbf{1}^{T} \overline{\mathbf{x}}_{m n}=1, \quad \forall m, n \in N, \quad(m, n) \in L
$$

where 1 denotes a $C \times 1$ vector with all entries equal to 1 . The term $\mathbf{1}^{T} \overline{\mathbf{x}}_{m n}$ is equal to 1 if node $m$ allocates one of the available frequency channels to communicate with node $n$.

Consider two logical links $(m, n),(p, q) \in L$. We have,

$$
\overline{\mathbf{x}}_{m n}^{T} \overline{\mathbf{x}}_{p q}= \begin{cases}1, & \begin{array}{l}
\text { if links }(m, n) \text { and }(p, q) \text { use } \\
\text { the same channel, } \\
\text { otherwise }
\end{array}\end{cases}
$$

\section{B. Interface Assignment Model}

For any two nodes $m, n \in N$ such that $(m, n) \in L$, we define an $I \times 1$ interface assignment vector $\overline{\mathbf{y}}_{m n}$. If the $i^{\text {th }}$ network interface in node $m$ is used to communicate with node $n$, then the $i^{\text {th }}$ element in $\overline{\mathbf{y}}_{m n}$ is equal to 1 ; otherwise, it is equal to zero. As an example, assume that $I=3$ and node $m$ assigns its first network interface to communicate with node $n$. We have, $\overline{\mathbf{y}}_{m n}=\left[\begin{array}{lll}1 & 0 & 0\end{array}\right]^{T}$.

To establish the logical link $(m, n) \in L$, routers $m$ and $n$ should allocate one of their NICs to communicate with each other. Note that in general $\overline{\mathbf{y}}_{m n} \neq \overline{\mathbf{y}}_{n m}$. However, it is still required that,

$$
\mathbf{1}^{T} \overline{\mathbf{y}}_{m n}=1, \quad \forall m, n \in N, \quad(m, n) \in L
$$

where 1 denotes an $I \times 1$ vector with all entries equal to 1 . The term $\mathbf{1}^{T} \overline{\mathbf{y}}_{m n}$ is equal to 1 if node $m$ assigns one of its NICs to communicate with node $n$.

Consider two logical links $(m, n),(m, p) \in L$ such that they share node $m \in N$. We have,

$$
\overline{\mathbf{y}}_{m n}^{T} \overline{\mathbf{y}}_{m p}= \begin{cases}1, & \text { if links }(m, n) \text { and }(m, p) \text { share } \\ & \text { an NIC on node } m, \\ 0, & \text { otherwise. }\end{cases}
$$

\section{Joint Channel Allocation and Interface Assignment}

If two neighboring logical links $(m, n),(m, p) \in L$ share an NIC on node $m$ (i.e., $\overline{\mathbf{y}}_{m n}^{T} \overline{\mathbf{y}}_{m p}=1$ ), then they will be assigned the same frequency channel (i.e., $\overline{\mathbf{x}}_{m n}^{T} \overline{\mathbf{x}}_{m p}=1$ ). On the other hand, if they are not sharing an NIC (i.e., $\overline{\mathbf{y}}_{m n}^{T} \quad \overline{\mathbf{y}}_{m p}=0$ ), they will be assigned to two different frequency channels (i.e., $\overline{\mathbf{x}}_{m n}^{T} \overline{\mathbf{x}}_{m p}=0$ ). In this paper, we assume that $C \geq I$. We can model the relationship between the channel allocation and the interface assignment vectors to be as follows:

$\overline{\mathbf{x}}_{m n}^{T} \overline{\mathbf{x}}_{m p}=\overline{\mathbf{y}}_{m n}^{T} \overline{\mathbf{y}}_{m p}, \quad \forall m, n, p \in N, \quad(m, n),(m, p) \in L$

A joint channel allocation and interface assignment strategy, denoted by $\langle\overline{\mathbf{x}}, \overline{\mathbf{y}}\rangle$, is defined as determining the channel allocation vector $\overline{\mathbf{x}}_{m n}$ and the interface assignment vector $\overline{\mathbf{y}}_{m n}$ for all logical links $(m, n) \in L$. 
Given an MC-WMN logical topology, a joint channel allocation and interface assignment strategy $\langle\overline{\mathbf{x}}, \overline{\mathbf{y}}\rangle$ is feasible, if for all $m, n, p \in N$ and $(m, n),(m, p) \in L$, we have:

$$
\begin{gathered}
\overline{\mathbf{x}}_{m n}, \overline{\mathbf{x}}_{m p} \in\{0,1\}^{C}, \\
\overline{\mathbf{y}}_{m n}, \overline{\mathbf{y}}_{m p} \in\{0,1\}^{I}, \\
\overline{\mathbf{x}}_{m n}=\overline{\mathbf{x}}_{n m}, \\
\mathbf{1}^{T} \overline{\mathbf{x}}_{m n}=1, \\
\mathbf{1}^{T} \overline{\mathbf{y}}_{m n}=1, \\
\overline{\mathbf{x}}_{m n}^{T} \overline{\mathbf{x}}_{m p}=\overline{\mathbf{y}}_{m n}^{T} \overline{\mathbf{y}}_{m p}
\end{gathered}
$$

where $\{0,1\}^{C}$ and $\{0,1\}^{I}$ denote the set of all $C \times 1$ and $I \times 1$ binary vectors, respectively. The set of all feasible joint channel allocation and interface assignment strategies is denoted by $\Psi$.

\section{Interference Model}

In an MC-WMN, two logical links $(m, n),(p, q) \in L$ are defined to be mutually interfered with each other whenever both of the following conditions hold:

1) The logical links operate over the same frequency channel (i.e., $\overline{\mathbf{x}}_{m n}^{T} \overline{\mathbf{x}}_{p q}=1$ ), and

2) The sender/receiver of one link is within the interference range of the sender/receiver of the other link.

To model the interference between the logical links in MCWMN, we construct a link-layer flow contention graph (or simply contention graph [21], [22]). In a contention graph, vertices correspond to the logical links. There is an edge between two vertices if the corresponding logical links mutually interfere with each other and cannot be active simultaneously. The contention graph depends on the allocated channels and assigned interfaces. Given $\langle\overline{\mathbf{x}}, \overline{\mathbf{y}}\rangle$, the corresponding contention graph is denoted by $C G_{\langle\overline{\mathbf{x}}, \overline{\mathbf{y}}\rangle}$.

Consider a specific joint channel allocation and interface assignment strategy that assigns frequency channels and network interface cards as follows:

$$
\begin{array}{ll}
\overline{\mathbf{x}}_{m n}=[1,0, \cdots, 0]^{T}, \forall m, n \in N, & (m, n) \in L \\
\overline{\mathbf{y}}_{m n}=[1,0, \cdots, 0]^{T}, \forall m, n \in N, & (m, n) \in L
\end{array}
$$

We can show that (8) is a feasible channel allocation and interface assignment strategy. It simply assigns all links to the first channel and all links in each node to the first NIC. The corresponding contention graph is called singlechannel contention graph and is denoted by $C G_{S}$. A sample wireless mesh network and its corresponding single-channel contention graph are depicted in Fig. 2. The communication and interference ranges are $100 \mathrm{~m}$ and $150 \mathrm{~m}$, respectively.

Although the vertices in $C G_{\langle\overline{\mathbf{x}}, \overline{\mathbf{y}}\rangle}$ and $C G_{S}$ are the same, $C G_{\langle\overline{\mathbf{x}}, \overline{\mathbf{y}}\rangle}$ may have less edges than $C G_{S}$. Thus,

$$
C G_{\langle\overline{\mathbf{x}}, \overline{\mathbf{y}}\rangle} \subseteq C G_{\boldsymbol{S}}, \quad \forall\langle\overline{\mathbf{x}}, \overline{\mathbf{y}}\rangle \in \Psi
$$

Given a $C G_{\langle\overline{\mathbf{x}}, \overline{\mathbf{y}}\rangle}$, we can identify all of its maximal cliques $^{2}$. The links corresponded to the vertices of a maximal clique cannot be active simultaneously [22]-[24]. Let $\mathbb{Q}\langle\overline{\mathbf{x}}, \overline{\mathbf{y}}\rangle$

\footnotetext{
${ }^{2} \mathrm{~A}$ maximal clique in a graph is a maximal complete subgraph of the graph.
}

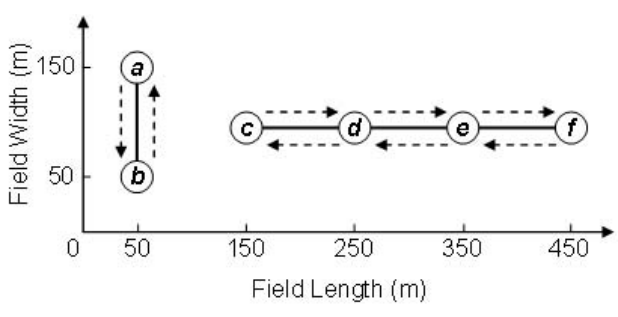

(a) Physical and logical topologies.

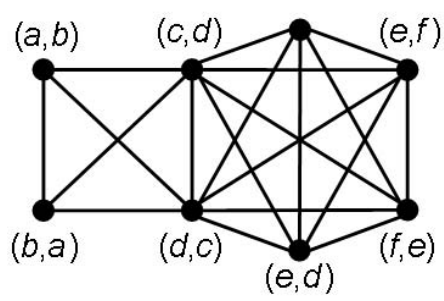

(b) Corresponding single-channel contention graph $C G_{\boldsymbol{S}}$.

Fig. 2. A sample wireless mesh network with 6 nodes and 8 links.

denote the set of all maximal cliques in $C G_{\langle\overline{\mathbf{x}}, \overline{\mathbf{y}}\rangle}$. The number of maximal cliques is denoted by $\left|\mathbb{Q}_{\langle\overline{\mathbf{x}}, \overline{\mathbf{y}}\rangle}\right|$. For notation simplicity, we enumerate the maximal cliques. The $i^{\text {th }}$ maximal clique of $C G_{\langle\overline{\mathbf{x}}, \overline{\mathbf{y}}\rangle}$ is denoted by $Q_{\langle\overline{\mathbf{x}}, \overline{\mathbf{y}}\rangle}^{i}$. The set of vertices that form $Q_{\langle\overline{\mathbf{x}}, \overline{\mathbf{y}}\rangle}^{i}$ is denoted by $V_{\langle\overline{\mathbf{x}}, \overline{\mathbf{y}}\rangle}^{i}$. Note that $V_{\langle\overline{\mathbf{x}}, \overline{\mathbf{y}}\rangle}^{i} \subseteq L$.

Let $f_{m n}>0$ denote the normalized link-layer flow rate on logical link $(m, n) \in L$ (i.e., the proportion of time that link $(m, n)$ is active). Since flows within the same maximal clique cannot transmit simultaneously, we have the following clique capacity constraint [22]-[24]:

$$
\sum_{p, q:(p, q) \in V_{\langle\overline{\mathbf{x}}, \overline{\mathbf{y}}\rangle}^{i}} f_{p q} \leq \varepsilon, \quad \forall i: Q_{\langle\overline{\mathbf{x}}, \overline{\mathbf{y}}\rangle}^{i} \in \mathbb{Q}_{\langle\overline{\mathbf{x}}, \overline{\mathbf{y}}\rangle}
$$

where $0<\varepsilon \leq 1$ is the clique capacity. The constraint in (10) guarantees that the normalized link-layer flow rates are feasible. The proper value of $\varepsilon$ depends on some specific characteristics of the graph $C G_{\langle\overline{\mathbf{x}}, \overline{\mathbf{y}}\rangle}$. Selecting $\varepsilon=1$ is a sufficient condition for the feasibility of the allocated linklayer flow rates if and only if $C G_{\langle\overline{\mathbf{x}}, \overline{\mathbf{y}}\rangle}$ is a perfect $\operatorname{graph}^{3}$. According to the strong perfect graph theorem [26], a graph is perfect if and only if it has no induced subgraph that is isomorphic to an odd hole ${ }^{4}$. If there exists odd holes in contention graph $C G_{\langle\bar{x}, \bar{y}\rangle}$, the clique capacity parameter $\varepsilon$ should be adequately reduced for any maximal clique that induces edges of an odd hole. For a general graph, it has been shown in [27] (also cited in [22], [28]) that if we set $\varepsilon=\frac{2}{3}$, then the feasibility of the allocated link-layer flow rates is guaranteed without having to check the characteristics of the contention graph. The proper selection of $\varepsilon$ can be studied further in [22]-[24], [28]. In this paper, we assume that $\varepsilon$ is a given design parameter.

\footnotetext{
${ }^{3} \mathrm{~A}$ graph is perfect if for every induced subgraph its chromatic number is equal to the clique number of the graph [25].

${ }^{4} \mathrm{~A}$ hole is a graph induced by a chordless cycle of length at least 4 . A hole is odd if it contains an odd number of vertices [26].
} 
To use clique capacity constraint (10) in a joint design problem, we need to find and express the maximal cliques of $C G_{\langle\bar{x}, \bar{y}\rangle}$ in terms of channel allocation and interface assignment vectors. This task is not easy in practice ${ }^{5}$. The following theorem provides an alternative approach.

Theorem 1. Given $\langle\overline{\mathbf{x}}, \overline{\mathbf{y}}\rangle \in \Psi$, the feasible region formed by constraint (10) is equivalent to the feasible region formed by the following constraint,

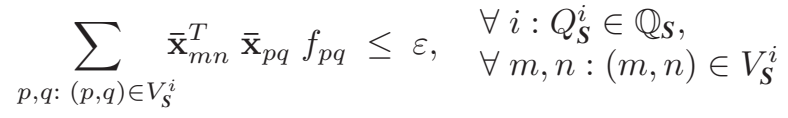

where $\mathbb{Q}_{\mathbf{S}}, Q_{\mathbf{S}}^{i}$, and $V_{\mathbf{S}}^{i}$ denote the set of maximal cliques, the $i^{\text {th }}$ maximal clique, and the set of vertices in the $i^{\text {th }}$ maximal clique of the single-channel contention graph $C G_{\mathbf{S}}$, respectively.

The proof of the above theorem is given in Appendix A. Note that the number of constraints in (10) and (11) are not equal. Depending on $\langle\overline{\mathbf{x}}, \overline{\mathbf{y}}\rangle$, the number of inequalities in (10) can vary from $\left|\mathbb{Q}_{S}\right|$ to $\frac{|L|}{2}$. On the other hand, the number of inequalities in (11) is fixed and is equal to $\sum_{i=1}^{\left|\mathbb{Q}_{S}\right|}\left|V_{S}^{i}\right|$. In addition, all the inequalities in (10) are maximal clique constraints; while there may be some inequalities in (11) that are just clique ${ }^{6}$ (but not maximal clique) constraints.

As an example, consider $C G_{S}$ in Fig. 2(b). Two maximal cliques are recognized: $V_{S}^{1}=\{(a, b),(b, a),(c, d),(d, c)\}$ and $V_{S}^{2}=\{(c, d),(d, c),(d, e),(e, d),(e, f),(f, e)\}$. They form $\left|V_{S}^{1}\right|+\left|V_{S}^{2}\right|=10$ inequalities in (11). The corresponding inequalities from $V_{S}^{1}$ are:

$$
\begin{aligned}
& \overline{\mathbf{x}}_{a b}^{T} \overline{\mathbf{x}}_{a b} f_{a b}+\overline{\mathbf{x}}_{a b}^{T} \overline{\mathbf{x}}_{b a} f_{b a}+\overline{\mathbf{x}}_{a b}^{T} \overline{\mathbf{x}}_{c d} f_{c d}+\overline{\mathbf{x}}_{a b}^{T} \overline{\mathbf{x}}_{d c} f_{d c} \leq \varepsilon \\
& \overline{\mathbf{x}}_{b a}^{T} \overline{\mathbf{x}}_{a b} f_{a b}+\overline{\mathbf{x}}_{b a}^{T} \overline{\mathbf{x}}_{b a} f_{b a}+\overline{\mathbf{x}}_{b a}^{T} \overline{\mathbf{x}}_{c d} f_{c d}+\overline{\mathbf{x}}_{b a}^{T} \overline{\mathbf{x}}_{d c} f_{d c} \leq \varepsilon \\
& \overline{\mathbf{x}}_{c d}^{T} \overline{\mathbf{x}}_{a b} f_{a b}+\overline{\mathbf{x}}_{c d}^{T} \overline{\mathbf{x}}_{b a} f_{b a}+\overline{\mathbf{x}}_{c d}^{T} \overline{\mathbf{x}}_{c d} f_{c d}+\overline{\mathbf{x}}_{c d}^{T} \overline{\mathbf{x}}_{d c} f_{d c} \leq \varepsilon \\
& \overline{\mathbf{x}}_{d c}^{T} \overline{\mathbf{x}}_{a b} f_{a b}+\overline{\mathbf{x}}_{d c}^{T} \overline{\mathbf{x}}_{b a} f_{b a}+\overline{\mathbf{x}}_{d c}^{T} \overline{\mathbf{x}}_{c d} f_{c d}+\overline{\mathbf{x}}_{d c}^{T} \overline{\mathbf{x}}_{d c} f_{d c} \leq \varepsilon
\end{aligned}
$$

If the first channel is assigned to links $(a, b),(b, a),(c, d)$, and $(d, c)$, then inequalities in (12) all become: $f_{a b}+f_{b a}+$ $f_{c d}+f_{d c} \leq \epsilon$. If the first channel is assigned to links $(a, b)$ and $(b, a)$ and the second channel is assigned to links $(c, d)$ and $(d, c)$, then inequalities (12a) and (12b) become: $f_{a b}+f_{b a} \leq \epsilon$, and inequalities (12c) and (12d) become: $f_{c d}+f_{d c} \leq \epsilon$.

\section{E. Joint Channel Allocation, Interface Assignment, and MAC Problem}

The mathematical formulation introduced in (1)-(11) models the relationship between the channel allocation, interface assignment, and MAC problems. The model can be used to develop different cross-layer algorithms in MC-WMNs. In this paper, we are interested in extending the fair MAC framework in [22] to obtain a joint channel allocation, interface assignment, and MAC algorithm.

\footnotetext{
${ }^{5}$ Finding the maximal cliques of an arbitrary graph is $N P$-hard [29].

${ }^{6} \mathrm{~A}$ clique of a graph is a complete subgraph of the graph. Each clique is either a maximal clique or a subgraph of a maximal clique.
}

The objective of a fair MAC algorithm is to find the feasible link-layer flow rates that satisfy some fairness properties [22]. Given an MC-WMN logical topology, $I$ network interface cards per router, $C$ orthogonal frequency channels, $C G_{S}$ and the set of its maximal cliques $\mathbb{Q}_{S}$, our objective is to choose the normalized link-layer flow rates, allocate channels, and assign interfaces, so as to solve the following constrained network utility maximization problem:

$$
\begin{array}{cll}
\max _{\substack{\langle>0,\langle\overline{\mathbf{x}}, \overline{\mathbf{y}}\rangle \in \Psi}} & \sum_{m, n:(m, n) \in L} U_{m n}\left(f_{m n}\right) & \\
\text { s.t. } & \sum_{p, q:} \overline{\mathbf{x}}_{m n}^{T} \overline{\mathbf{x}}_{p q} f_{p q} \leq \varepsilon, & \forall i: Q_{S}^{i} \in \mathbb{Q}_{S}, \\
& \forall m, n:(m, n) \in V_{S}^{i}
\end{array}
$$

where $U_{m n}$ is a continuously differentiable, increasing, and strictly concave utility function of the link-layer flow rate $f_{m n}$. The concavity of the objective function can guarantee fairness [30]. Some of the popular concave utility functions for proportional, max-min, and harmonic mean fairness are listed in [22] and [30].

\section{JOINT DESIGN I: EXACT BINARY LINEARIZATION}

The network utility maximization problem (13) is a nonlinear mixed-integer problem and is not easy to solve. Note that:

1) It has real variables $f$ and binary variables $\overline{\mathbf{x}}$ and $\overline{\mathbf{y}}$.

2) It has some mixed binary-real cubic and some pure binary quadratic constraints. The binary quadratic constraints appear in $\langle\overline{\mathbf{x}}, \overline{\mathbf{y}}\rangle \in \Psi$.

After relaxing the binary constraints, there is no guarantee that the cubic and quadratic constraints are convex. Thus, even the relaxed problem cannot be easily solved. In this section, we present some binary linearization techniques to obtain the global optimal solution of the network utility maximization problem (13) in a static and centralized manner.

Consider any logical links $(m, n),(p, q) \in L$. We denote $e_{p q}^{m n} \in C G_{S}$ if there is an edge between vertices $(m, n)$ and $(p, q)$ in graph $C G_{S}$. The polynomial constraints in (13) can be linearized by the following three steps:

Step 1: For each pair of logical links $(m, n),(p, q) \in L$ such that $e_{p q}^{m n} \in C G_{S}$, we define a $C \times 1$ auxiliary channel allocation vector $\overline{\mathbf{u}}_{p q}^{m n}$ as follows:

$$
\overline{\mathbf{u}}_{p q}^{m n}=\overline{\mathbf{x}}_{m n} \circ \overline{\mathbf{x}}_{p q}
$$

where $\circ$ denotes the Hadamard product ${ }^{7}$. From (14) we have, $\overline{\mathbf{x}}_{m n}^{T} \overline{\mathbf{x}}_{p q}=\mathbf{1}^{T} \overline{\mathbf{u}}_{p q}^{m n}$. Since $\overline{\mathbf{x}}_{m n}, \overline{\mathbf{x}}_{p q}$, and $\overline{\mathbf{u}}_{p q}^{m n}$ are $C \times 1$ binary vectors, eq. (14) is equivalent to the following linear constraint (see Appendix B):

$$
\begin{array}{r}
\overline{\mathbf{x}}_{m n}+\overline{\mathbf{x}}_{p q}-\overline{\mathbf{u}}_{p q}^{m n} \leq \mathbf{1}, \\
-\overline{\mathbf{x}}_{m n}-\overline{\mathbf{x}}_{p q}+2 \overline{\mathbf{u}}_{p q}^{m n} \leq \mathbf{0}
\end{array}
$$

\footnotetext{
${ }^{7}$ Hadamard product of two $C \times 1$ vectors $a$ and $b$ is a $C \times 1$ vector whose $i^{\text {th }}$ entry is equal to the product of the $i^{\text {th }}$ entry of $a$ and the $i^{\text {th }}$ entry of $b$ [31].
} 
Step 2: For each pair of logical links $(m, n),(m, p) \in L$ (they share node $m$ ), we define an $I \times 1$ auxiliary interface assignment vector $\overline{\mathbf{v}}_{m p}^{m n}$ to be as follows:

$$
\overline{\mathbf{v}}_{m p}^{m n}=\overline{\mathbf{y}}_{m n} \circ \overline{\mathbf{y}}_{m p}
$$

From (16) we have, $\overline{\mathbf{y}}_{m n}^{T} \overline{\mathbf{y}}_{m p}=\mathbf{1}^{T} \overline{\mathbf{v}}_{m p}^{m n}$. Eq. (16) is equivalent to the following constraint (see Appendix B):

$$
\begin{array}{r}
\overline{\mathbf{y}}_{m n}+\overline{\mathbf{y}}_{m p}-\overline{\mathbf{v}}_{m p}^{m n} \leq \mathbf{1}, \\
-\overline{\mathbf{y}}_{m n}-\overline{\mathbf{y}}_{m p}+2 \overline{\mathbf{v}}_{m p}^{m n} \leq \mathbf{0}
\end{array}
$$

A linearized joint channel allocation and interface assignment strategy, denoted by $\langle\overline{\mathbf{x}}, \overline{\mathbf{y}}, \overline{\mathbf{u}}, \overline{\mathbf{v}}\rangle$, is defined as determining $\overline{\mathbf{x}}_{m n}$ and $\overline{\mathbf{y}}_{m n}$ for all links $(m, n) \in L, \overline{\mathbf{u}}_{p q}^{m n}$ for all links $(m, n),(p, q) \in L$ such that $e_{p q}^{m n} \in C G_{\boldsymbol{S}}$, and $\overline{\mathbf{v}}_{m p}^{m n}$ for all links $(m, n),(m, p) \in L$. A linearized joint strategy $\langle\overline{\mathbf{x}}, \overline{\mathbf{y}}, \overline{\mathbf{u}}, \overline{\mathbf{v}}\rangle$ is feasible if for all $m, n, p, q \in N$ and $(m, n),(m, p),(p, q) \in L$ such that $e_{p q}^{m n} \in C G_{S}$, we have:

$$
\begin{gathered}
\overline{\mathbf{x}}_{m n}, \overline{\mathbf{x}}_{p q}, \overline{\mathbf{u}}_{p q}^{m n} \in\{0,1\}^{C}, \\
\overline{\mathbf{y}}_{m n}, \overline{\mathbf{y}}_{m p}, \overline{\mathbf{v}}_{m p}^{m n} \in\{0,1\}^{I}, \\
\overline{\mathbf{x}}_{m n}=\overline{\mathbf{x}}_{m m}, \\
\mathbf{1}^{T} \overline{\mathbf{x}}_{m n}=1, \\
\mathbf{1}^{T} \overline{\mathbf{y}}_{m n}=1, \\
\mathbf{1}^{T} \overline{\mathbf{u}}_{m p}^{m n}=\mathbf{1}^{T} \overline{\mathbf{v}}_{m p}^{m n}, \\
\overline{\mathbf{x}}_{m n}+\overline{\mathbf{x}}_{p q}-\overline{\mathbf{u}}_{p q}^{m n} \leq \mathbf{1}, \\
-\overline{\mathbf{x}}_{m n}-\overline{\mathbf{x}}_{p q}+2 \overline{\mathbf{u}}_{m q}^{m n} \leq \mathbf{0}, \\
\overline{\mathbf{y}}_{m n}+\overline{\mathbf{y}}_{m p}-\overline{\mathbf{v}}_{m p}^{m n} \leq \mathbf{1}, \\
-\overline{\mathbf{y}}_{m n}-\overline{\mathbf{y}}_{m p}+2 \overline{\mathbf{v}}_{m p}^{m n} \leq \mathbf{0}
\end{gathered}
$$

where the linear equality $\mathbf{1}^{T} \overline{\mathbf{u}}_{m p}^{m n}=\mathbf{1}^{T} \overline{\mathbf{v}}_{m p}^{m n}$ in constraint (18) is equivalent to the nonlinear equality $\overline{\mathbf{x}}_{m n}^{T} \overline{\mathbf{x}}_{m p}=\overline{\mathbf{y}}_{m n}^{T} \overline{\mathbf{y}}_{m p}$ in constraint (7). The set of all feasible linearized joint channel allocation and interface assignment strategies is denoted by $\Phi$.

Step 3: For each pair of logical links $(m, n),(p, q) \in L$ such that $e_{p q}^{m n} \in C G_{S}$, we define an auxiliary real scalar $z_{p q}^{m n}$ to be as follows:

$$
z_{p q}^{m n}=\overline{\mathbf{x}}_{m n}^{T} \overline{\mathbf{x}}_{p q} f_{p q}=\left(\mathbf{1}^{T} \overline{\mathbf{u}}_{p q}^{m n}\right) f_{p q}
$$

Since $\mathbf{1}^{T} \overline{\mathbf{u}}_{p q}^{m n}$ is a binary scalar and the upper bound for the normalized link-layer flow $f_{p q}$ is equal to one, eq. (19) is equivalent to the following constraint (see Appendix B):

$$
\begin{aligned}
& 0 \leq z_{p q}^{m n} \leq f_{p q} \\
& f_{p q}-1+\mathbf{1}^{T} \overline{\mathbf{u}}_{p q}^{m n} \leq z_{p q}^{m n} \leq \mathbf{1}^{T} \overline{\mathbf{u}}_{p q}^{m n}
\end{aligned}
$$

Combining steps 1-3, the global utility maximization problem (13) is equivalent to the following problem:

$$
\begin{aligned}
& \max _{f>0,} \sum_{m, n:(m, n) \in L} U_{m n}\left(f_{m n}\right) \\
& \begin{array}{l}
f>0 \\
z \geq 0
\end{array} \\
& \langle\overline{\mathbf{x}}, \overline{\mathbf{y}}, \overline{\overline{\mathbf{u}}}, \overline{\mathbf{v}}\rangle \in \Phi \\
& \text { s.t. } \quad \sum_{p, q:(p, q) \in V_{s}^{i}} z_{p q}^{m n} \leq \varepsilon \\
& z_{p q}^{m n} \leq f_{p q}, \\
& f_{p q}-1+\mathbf{1}^{T} \overline{\mathbf{u}}_{p q}^{m n} \leq z_{p q}^{m n}, \\
& z_{p q}^{m n} \leq \mathbf{1}^{T} \overline{\mathbf{u}}_{p q}^{m n}, \\
& \forall i: Q_{S}^{i} \in \mathbb{Q}_{S}, \\
& \forall m, n:(m, n) \in V_{S}^{i} \\
& \forall m, n, p, q: e_{p q}^{m n} \in C G_{S} \\
& \forall m, n, p, q: e_{p q}^{m n} \in C G_{S} \\
& \forall m, n, p, q: e_{p q}^{m n} \in C G_{S}
\end{aligned}
$$

By relaxing the binary constraints on $\overline{\mathbf{x}}, \overline{\mathbf{y}}, \overline{\mathbf{u}}$, and $\overline{\mathbf{v}},(21)$ becomes a strictly concave problem with a unique global maximum. There exist several efficient algorithms to solve concave maximization problems [32]. Solution of the relaxed problem can be used to obtain the required bounds for the branch and bound algorithm [33] to achieve one of the global optimums of the original mixed-integer problem (21). In general, problem (21) may have multiple global maxima.

\section{Joint DESIGN II: Approximate DuAL DECOMPOSITION}

The proposed exact binary linearization scheme in Section III can lead to obtaining the global optimal solution of the joint channel allocation, interface assignment, and MAC problem (13) in a static and centralized manner. In this section, by making some assumptions, we propose an alternative but approximate design which is of more interest in terms of practical deployment.

Consider the following dual problem of the original primal problem (13):

$$
\min _{\rho \geq 0} D(\rho)
$$

with partial dual function

$$
\begin{aligned}
& D(\rho)= \\
& \quad \underset{\substack{f>0,\langle\overline{\mathbf{x}}, \overline{\mathbf{y}}\rangle \in \Psi}}{ } \sum_{m, n:(m, n) \in L} U_{m n}\left(f_{m n}\right)- \\
& \quad \sum_{i=1}^{\left|\mathbb{Q}_{s}\right|} \sum_{m, n:(m, n) \in V_{s}^{i}} \rho_{m n}^{i}\left(\sum_{p, q:(p, q) \in V_{s}^{i}} \overline{\mathbf{x}}_{m n}^{T} \overline{\mathbf{x}}_{p q} f_{p q}-\varepsilon\right)
\end{aligned}
$$

where we relaxed the clique capacity constraint in (13). The Lagrangian multiplier for the clique capacity constraint corresponding to clique $Q_{S}^{i} \in \mathbb{Q}_{S}$ and vertex $(m, n) \in V_{S}^{i}$ is denoted by $\rho_{m n}^{i}$. We make the following assumptions:

1) The normalized link-layer flow rates are updated every $T_{M A C}$ time units.

2) The allocated channels and assigned interfaces are updated every $T_{C I}$ time units.

3) The time interval $T_{M A C} \ll T_{C I}$.

We now decompose $D(\rho)$ approximately into the following two subproblems:

$$
\begin{aligned}
& D_{1}(\rho,\langle\overline{\mathbf{x}}, \overline{\mathbf{y}}\rangle)= \\
& \max _{f>0} \sum_{m, n:(m, n) \in L} U_{m n}\left(f_{m n}\right)- \\
& \quad \sum_{i=1}^{\left|\mathbb{Q}_{s}\right|} \sum_{m, n:(m, n) \in V_{s}^{i}} \rho_{m n}^{i} \sum_{p, q:(p, q) \in V_{s}^{i}}\left(\overline{\mathbf{x}}_{m n}^{T} \overline{\mathbf{x}}_{p q}\right) f_{p q}
\end{aligned}
$$

and

$$
\begin{aligned}
& D_{2}(\rho, f)=- \\
& \max _{\langle\overline{\mathbf{x}}, \overline{\mathbf{y}}\rangle \in \Psi} \sum_{i=1}^{\left|\mathbb{Q}_{s}\right|} \sum_{m, n:(m, n) \in V_{s}^{i}} \rho_{m n}^{i} \sum_{p, q:(p, q) \in V_{s}^{i}}\left(\overline{\mathbf{x}}_{m n}^{T} \overline{\mathbf{x}}_{p q}\right) f_{p q}
\end{aligned}
$$

The first subproblem is just a fair MAC [22], [34] over fixed channels and fixed interfaces. It is solved every $T_{M A C}$ time units. The term $\langle\overline{\mathbf{x}}, \overline{\mathbf{y}}\rangle$ in $D_{1}(\rho,\langle\overline{\mathbf{x}}, \overline{\mathbf{y}}\rangle)$ denotes the most recent obtained solution of (25) at the time that (24) is being 
solved. The second subproblem is a joint channel allocation and interface assignment algorithm. It is solved every $T_{C I}$ time units. The term $f$ in $D_{2}(\rho, f)$ denotes the most recent obtained solution of (24) at the time that (25) is being solved. Since $T_{C I}$ is assumed to be large enough, the link-layer flow rates converge to their corresponding optimal values before the next time that the channels and interfaces are being updated [22]. When we update the allocated channels and assigned interfaces in subproblem (25), the converged link-layer flow rates are assumed to be constant.

Using the approximate subgradient method [32], [35], the Lagrangian multiplier $\rho_{m n}^{i}$ is adjusted every $T_{M A C}$ time units as follows:

$$
\begin{aligned}
& \rho_{m n}^{i}(t+1)=\left[\rho_{m n}^{i}(t)+\right. \\
& \left.\xi_{\rho}(t)\left(\sum_{p, q:(p, q) \in V_{S}^{i}} \overline{\mathbf{x}}_{m n}^{T} \overline{\mathbf{x}}_{p q} f_{p q}(\rho(t),\langle\overline{\mathbf{x}}, \overline{\mathbf{y}}\rangle)-\varepsilon\right)\right]^{+}
\end{aligned}
$$

where $\xi_{\rho}(t)$ is a positive scalar stepsize and $f(\rho(t),\langle\overline{\mathbf{x}}, \overline{\mathbf{y}}\rangle)$ denotes the maximizer of (24) at time $t \geq 0$. We also denote: $[X]^{+}=\max (X, 0)$. The Lagrangian multipliers can be interpreted as clique contention prices to regulate between the supply and demand. Eq. (26) says that, if the demand $\sum_{(p, q) \in V_{s}^{i}} \overline{\mathbf{x}}_{m n}^{T} \overline{\mathbf{x}}_{p q} f_{p q}(\rho(t),\langle\overline{\mathbf{x}}, \overline{\mathbf{y}}\rangle)$ exceeds the supply $\varepsilon$, the price $\rho_{m n}^{i}$ will increase.

Lemma 1. Let $Q_{S}^{i}$ and $Q_{S}^{j}$ be two arbitrary maximal cliques in $C G_{S}$. For the logical links $(m, n),(p, q) \in L$, we have: $(m, n) \in V_{S}^{i}$ and $(p, q) \in V_{S}^{j}$. Given the allocated channels and assigned interfaces $\langle\overline{\mathbf{x}}, \overline{\mathbf{y}}\rangle$, if

$$
V_{S}^{i} \backslash\left\{(l, k): \overline{\mathbf{x}}_{m n}^{T} \overline{\mathbf{x}}_{l k}=0\right\} \subset V_{S}^{j} \backslash\left\{(l, k): \overline{\mathbf{x}}_{p q}^{T} \overline{\mathbf{x}}_{l k}=0\right\}
$$

then

$$
\sum_{l, k:(l, k) \in V_{s}^{i}} \overline{\mathbf{x}}_{m n}^{T} \overline{\mathbf{x}}_{l k} f_{l k}<\sum_{l, k:(l, k) \in V_{s}^{j}} \overline{\mathbf{x}}_{p q}^{T} \overline{\mathbf{x}}_{l k} f_{l k}
$$

and

$$
\lim _{t \rightarrow \infty} \rho_{m n}^{i}(t)=0
$$

which means that the clique contention prices converge to zero for those cliques that are not maximal cliques for $C G_{\langle\overline{\mathbf{x}}, \overline{\mathbf{y}}\rangle}$.

Lemma 2. For arbitrary logical links $(m, n),(p, q) \in L$, if $\overline{\mathbf{x}}_{m n}^{T} \overline{\mathbf{x}}_{p q}=1$ and $\rho_{m n}^{i}(0)=\rho_{p q}^{i}(0)$, then

$$
\rho_{m n}^{i}(t)=\rho_{p q}^{i}(t), \quad \forall i:(m, n),(p, q) \in V_{\boldsymbol{S}}^{i}, \forall t \geq 0
$$

The proofs of Lemma 1 and 2 are given in Appendix C. Note that by definition we have, $\overline{\mathbf{x}}_{m n}^{T} \overline{\mathbf{x}}_{p q}=\overline{\mathbf{x}}_{p q}^{T} \overline{\mathbf{x}}_{m n}$. Thus, the maximization in (25) is equivalent to:

$\left.\min _{\langle\overline{\mathbf{x}}, \overline{\mathbf{y}}\rangle \in \Psi} \sum_{i=1}^{\left|\mathbb{Q}_{s}\right|} \sum_{m, n, p, q:(m, n),(p, q) \in V_{s}^{i}} \frac{\rho_{m n}^{i} f_{p q}+\rho_{p q}^{i} f_{m n}}{2}\right) \overline{\mathbf{x}}_{m n}^{T} \overline{\mathbf{x}}_{p q}$

For logical links $(m, n),(p, q) \in L$, we define $\alpha_{p q}^{m n}$ to be:

$$
\begin{aligned}
\alpha_{p q}^{m n} & =\sum_{i:(m, n),(p, q) \in V_{s}^{i}}\left(\rho_{m n}^{i} f_{p q}+\rho_{p q}^{i} f_{m n}\right) \\
& =\left(f_{m n}+f_{p q}\right)\left(\sum_{i:(m, n),(p, q) \in V_{s}^{i}} \rho_{m n}^{i}\right) \\
& =\left(f_{p q}+f_{m n}\right)\left(\sum_{i:(m, n),(p, q) \in V_{s}^{i}} \rho_{p q}^{i}\right)=\alpha_{m n}^{p q}
\end{aligned}
$$

where the second and the third equalities result from Lemma 2. From (31) and (32), the joint channel allocation and interface assignment subproblem $D_{2}$ becomes:

$$
\min _{\langle\overline{\mathbf{x}}, \overline{\mathbf{y}}\rangle \in \Psi} \frac{1}{2} \sum_{m, n, p, q:(m, n),(p, q) \in L} \alpha_{p q}^{m n}\left(\overline{\mathbf{x}}_{m n}^{T} \overline{\mathbf{x}}_{p q}\right)
$$

where $\alpha_{p q}^{m n}$ is interpreted as the interference cost of having the logical links $(m, n)$ and $(p, q)$ be operated over the same frequency channel. From (32), the interference cost is high if the links are highly loaded and belong to highly contended maximal cliques. The quadratic binary program (33) minimizes the aggregated interference cost across the whole network. Using steps 1 and 2 in Section III, program (33) is transformed to the following equivalent linear binary program:

$$
\min _{\langle\overline{\mathbf{x}}, \overline{\mathbf{y}}, \overline{\mathbf{u}}, \overline{\mathbf{v}}\rangle \in \Phi} \frac{1}{2} \sum_{m, n, p, q:(m, n),(p, q) \in L} \alpha_{p q}^{m n}\left(\mathbf{1}^{T} \overline{\mathbf{u}}_{p q}^{m n}\right)
$$

There are effective commercial computer codes (such as CPLEX [36]) to solve the linear binary problems. Here, we assume that all the required information to solve (34) is gathered every $T_{C I}$ time units in a pre-authorized node (e.g., one of the gateways). The pre-authorized node solves the linearized problem and announces the optimal channels and interfaces to the whole network. If $T_{C I}$ is large enough, the signalling overhead to implement (34) is not significant. In practice, the channel allocation and interface assignment interval $T_{C I}$ can be in the order of several minutes [3], [12].

Because of the approximate dual decomposition, the optimality of the joint algorithms in (24), (26), and (34) is not guaranteed. We will investigate the sub-optimality of the solutions in Section V.

\section{NUMERICAL EXAMPLES}

In this section, we evaluate the characteristics of our proposed joint channel allocation, interface assignment, and MAC designs. In the studied model, the size of the network field is $300 \mathrm{~m} \times 200 \mathrm{~m}$. Ten different random scenarios are considered. In each scenario, the WMN consists of ten wireless mesh routers that are arbitrarily located in the field. The routers are equipped with two NICs (i.e., $I=2$ ) and three orthogonal frequency channels are being used (i.e., $C=3$ ). The communication and interference ranges are $100 \mathrm{~m}$ and $150 \mathrm{~m}$, respectively. For each scenario, there is a logical link between each pair of nodes if they are within the communication range of each other. One of the network scenarios we used in our numerical analysis is shown in Fig. 3.

The utility function is selected to be $U_{m n}\left(f_{m n}\right)=$ $\log \left(f_{m n}\right)$ for each logical link $(m, n) \in L$. Note that the logarithmic utility function leads to proportional fairness among the link-layer flow rates [22]. The stepsize $\xi_{\rho}$ is set to 0.1 . Clique capacity parameter $\varepsilon$ is equal to 1 , unless otherwise is specified. For the second joint design scheme, we have: $T_{M A C}=1$ and $T_{C I}=1000$. Note that, depending on the selected value for the stepsize $\xi_{\rho}$, the channel/interface update interval $T_{C I}$ should be large enough to let the fair MAC reaches its steady state. All NICs initially (at time $t=0$ ) are assigned to a single channel. 
TABLE I

NUMERICAL RESULTS FOR TEN DIFFERENT RANDOM SCENARIOS.

\begin{tabular}{|c|c|c|c|c|c|c|}
\hline \multirow{2}{*}{$\begin{array}{c}\text { Scenario } \\
\text { Number }\end{array}$} & \multicolumn{3}{|c|}{ Network Utility } & \multicolumn{3}{c|}{ Network Throughput } \\
\cline { 2 - 7 } & $\mathbf{D 1}^{\dagger}$ & $\mathbf{D 2}^{\ddagger}$ & SC $^{\S}$ & $\mathbf{D 1}$ & $\mathbf{D 2}$ & $\mathbf{S C}$ \\
\hline $\mathbf{1}$ & -40.54 & -40.54 & -66.71 & 52.3 & 52.3 & 17.4 \\
\hline $\mathbf{2}$ & -31.88 & -31.88 & -55.79 & 63.2 & 63.2 & 21.0 \\
\hline $\mathbf{3}$ & -46.09 & -46.09 & -72.45 & 41.3 & 41.3 & 13.8 \\
\hline $\mathbf{4}$ & -25.29 & -25.66 & -44.39 & 51.4 & 51.3 & 18.4 \\
\hline $\mathbf{5}$ & -35.36 & -35.36 & -61.73 & 66.0 & 66.0 & 22.0 \\
\hline $\mathbf{6}$ & -24.95 & -24.95 & -46.05 & 63.4 & 63.4 & 21.8 \\
\hline $\mathbf{7}$ & -35.02 & -35.02 & -57.04 & 55.0 & 55.0 & 23.9 \\
\hline $\mathbf{8}$ & -27.39 & -27.39 & -45.30 & 44.0 & 44.0 & 17.2 \\
\hline $\mathbf{9}$ & -35.70 & -35.70 & -59.69 & 52.3 & 52.3 & 18.1 \\
\hline $\mathbf{1 0}$ & -29.11 & -29.11 & -52.90 & 66.0 & 66.0 & 22.0 \\
\hline Average & $\mathbf{- 3 3 . 1 4}$ & $\mathbf{- 3 3 . 1 7}$ & $\mathbf{- 5 6 . 2 1}$ & $\mathbf{5 5 . 4 9}$ & $\mathbf{5 5 . 4 8}$ & $\mathbf{1 9 . 5 6}$ \\
\hline
\end{tabular}

$\dagger$ Design I: Exact Binary Linearization (from Section III)

$\ddagger$ Design II: Approximate Dual Decomposition (from Section IV)

$\S$ Single Channel

To evaluate the performance, two metrics are considered: 1 ) network utility, 2) network throughput. The former is the value of the objective function in the primal joint problem (13). The latter is the aggregate link-layer flow rate across all logical links. That is, $\sum_{m, n:(m, n) \in L} \kappa f_{m n}$. Parameter $\kappa$ denotes the nominal link-layer data rate in bits per second. Therefore, the term $\kappa f_{m n}$ denotes the actual link-layer flow rate on logical link $(m, n)$. Here we assume that $\kappa=11$ Mbps.

The numerical results for ten different random scenarios are listed in Table I. It is observed that both design schemes achieve significantly higher network throughput comparing to the single channel case. In addition, the second design scheme can lead to finding the optimal solutions in nine out of ten scenarios. Only in one scenario (i.e., scenario number four), a near-optimal solution (with $98.5 \%$ optimality) is attained. Note that, since the normalized link-layer flow rates are always less than or equal to one, the logarithmic network utility is negative-valued and culminates at zero.

Fig. 4 shows the trends of the network utility as well as the normalized link-layer flow rates $f_{a b}, f_{d i}$, and $f_{g h}$ for scenario number 1 . We can see that, after only two channel/interface update intervals, the network utility approaches its optimal value. Later on, after the eighth channel/interface update interval, the exact global optimal solution is reached and the allocated channels and assigned interfaces remain unchanged.

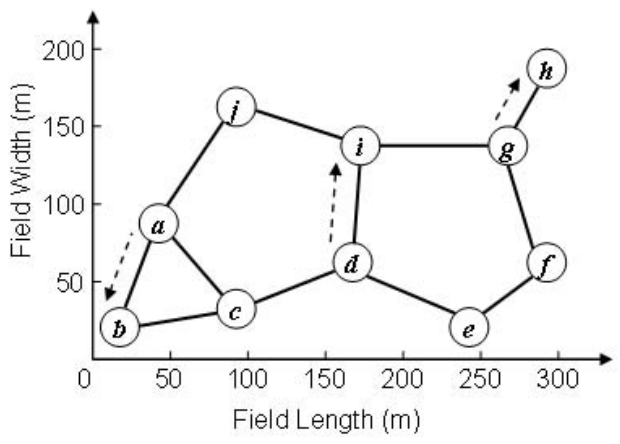

Fig. 3. Scenario number 1: a wireless mesh network with 10 nodes and 24 logical links.

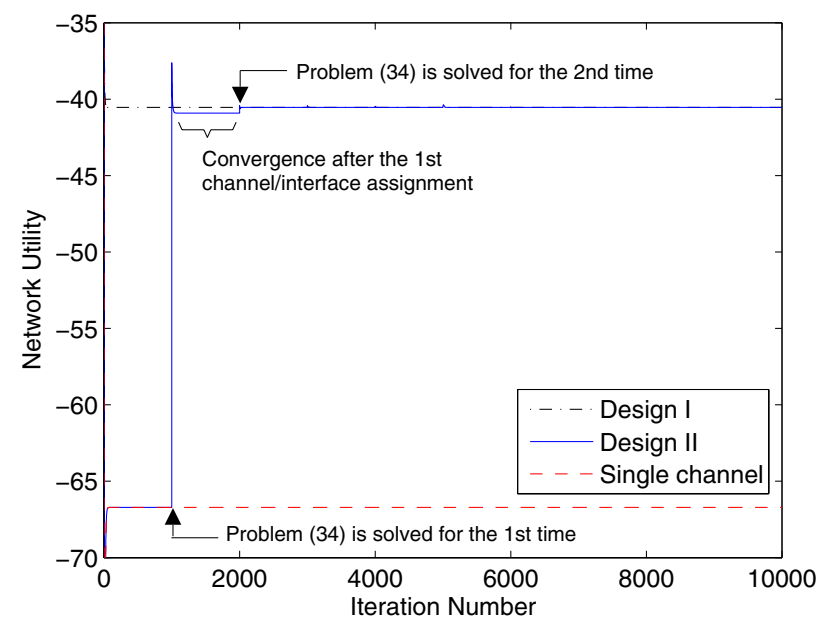

(a) Network utility

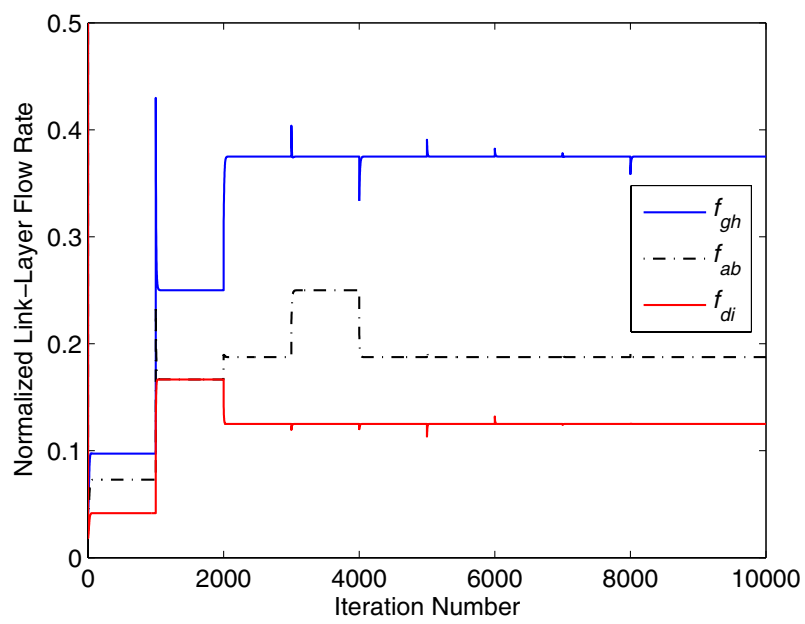

(b) Normalized link-layer flow rates for logical links $(a, b),(d, i)$, and $(g, h)$ when the second joint design scheme is applied.

Fig. 4. Trends for scenario number 1.

As stated in Section II, the proper value for the clique capacity parameter $\varepsilon$ should be selected depending on some specific characteristics of the network contention graph. In this experiment, we show that our proposed schemes are efficient, regardless of the selected value for parameter $\varepsilon$. Fig. 5 shows the average network utility as the clique capacity parameter $\varepsilon$ changes. In this figure, each point is the average of the measurements for all ten scenarios. It is observed that the multi-channel multi-interface deployment significantly increases the network average utility for all different values of $\epsilon$. In addition, our second proposed joint design efficiently finds the optimal or near-optimal solutions.

\section{CONClusions And Future Work}

We present a formulation for joint channel allocation, interface assignment, and MAC problem. We first model the channel allocation and interface assignment problems as well as their relationship by introducing binary channel allocation 


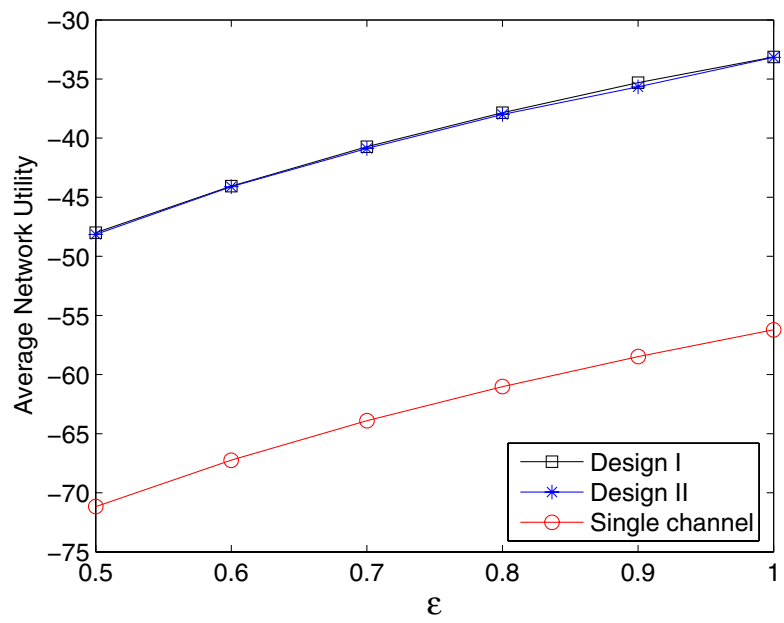

Fig. 5. Effects of varying the clique capacity parameter $\epsilon$.

and binary interface assignment vectors. We then obtain the feasible region of the link-layer flow rates as a function of the binary channel allocation and interface assignment vectors. The joint problem is then formulated as a cross-layer nonlinear mixed-integer utility maximization. An optimal joint design, based on exact binary linearization techniques, is proposed which leads to a global maximum. A near-optimal joint design, based on approximate dual decomposition techniques, is also proposed which is more practical for implementation.

This paper is a preliminary step towards a systematic approach to solve the channel allocation and interface assignment problems jointly with other network management algorithms using a network utility maximization framework. For future work, we plan to extend the model to a joint channel allocation, interface assignment, MAC, and congestion control design by replacing the link-layer utility maximization problem in (13) by a transport-layer utility maximization problem (e.g., [21], [37]). We shall also extend the model to assign not only the orthogonal channels but also the nonorthogonal (i.e., partially overlapped) channels [38] in order to utilize the available frequency spectrum more efficiently.

\section{APPENDIX}

\section{A. Proof of Theorem 1}

Since (10) includes all maximal clique capacity constraints for $C G_{\langle\bar{x}, \bar{y}\rangle}$ and each inequality in (11) is a clique (not necessarily a maximal clique) capacity constraint for $C G_{\langle\overline{\mathbf{x}}, \overline{\mathbf{y}}\rangle}$, then the feasible region formed by (10) is a subset of or equal to the feasible set formed by (11). We only need to prove that the reverse is also true. That is, the feasible region formed by (11) is a subset of or equal to the feasible region formed by (10). From (9) we have:

$$
\forall Q_{\langle\overline{\mathbf{x}}, \overline{\mathbf{y}}\rangle}^{i} \in \mathbb{Q}_{\langle\overline{\mathbf{x}}, \overline{\mathbf{y}}\rangle} \Rightarrow \exists Q_{S}^{j} \in \mathbb{Q}_{S}: Q_{\langle\overline{\mathbf{x}}, \overline{\mathbf{y}}\rangle}^{i} \subseteq Q_{S}^{j}
$$

We refer to $Q_{S}^{j}$ as the parent of $Q_{\langle\overline{\mathbf{x}}, \overline{\mathbf{y}}\rangle}^{i}$. In general, there may be more than one parent for $Q_{\langle\overline{\mathbf{x}}, \overline{\mathbf{y}}\rangle}^{i}\langle$
Consider an arbitrary maximal clique $Q_{\langle\overline{\mathbf{x}}, \overline{\mathbf{y}}\rangle}^{i}$ and one of its parents $Q_{S}^{j}$. Let $(m, n)$ be a logical link in $Q_{\langle\overline{\mathbf{x}}, \overline{\mathbf{y}}\rangle}^{i}$, that is: $(m, n) \in V_{\langle\overline{\mathbf{x}}, \overline{\mathbf{y}}\rangle}^{i}$. We can show (by contradiction) that,

$$
\begin{aligned}
& \overline{\mathbf{x}}_{m n}^{T} \overline{\mathbf{x}}_{p q}=1, \quad \forall p, q:(p, q) \in V_{\langle\overline{\mathbf{x}}, \overline{\mathbf{y}}\rangle}^{i} \\
& \overline{\mathbf{x}}_{m n}^{T} \overline{\mathbf{x}}_{p q}=0, \quad \forall p, q:(p, q) \in V_{\boldsymbol{S}}^{j} \backslash V_{\langle\overline{\mathbf{x}}, \overline{\mathbf{y}}\rangle}^{i}
\end{aligned}
$$

Thus, we have:

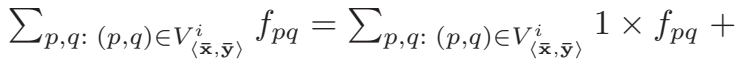

$$
\begin{aligned}
& \sum_{p, q:(p, q) \in V_{S}^{j} \backslash V_{\langle\overline{\mathbf{x}}, \overline{\mathbf{y}}\rangle}^{i}} 0 \times f_{p q} \\
& =\sum_{p, q:(p, q) \in V_{\langle\overline{\mathbf{x}}, \overline{\mathbf{y}}\rangle}^{i}} \overline{\mathbf{x}}_{m n}^{T} \overline{\mathbf{x}}_{p q} f_{p q}+
\end{aligned}
$$

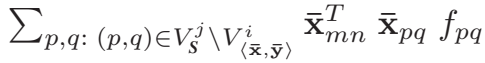

$$
\begin{aligned}
& =\sum_{p, q:(p, q) \in V_{s}^{j}} \overline{\mathbf{x}}_{m n}^{T} \overline{\mathbf{x}}_{p q} f_{p q}
\end{aligned}
$$

where the second equality follows from (36). Eq. (37) implies that for every inequality in (10), there is an equivalent inequality in (11). Therefore, the feasible region formed by (11) is a subset of or equal to the one formed by (10).

\section{B. Linearization Techniques}

Consider two binary variables $\theta_{1}$ and $\theta_{2}$. Their product (i.e., the quadratic term $\theta_{1} \theta_{2}$ ) can be replaced by a new binary auxiliary variable $\pi$, such that its values correspond to the values of $\theta_{1}$ and $\theta_{2}$ as follows:

$$
\pi= \begin{cases}0 & \text { if } \theta_{1}=0, \theta_{2}=0 \\ 0 & \text { if } \theta_{1}=0, \theta_{2}=1 \\ 0 & \text { if } \theta_{1}=1, \theta_{2}=0 \\ 1 & \text { if } \theta_{1}=1, \theta_{2}=1\end{cases}
$$

The desired correspondence is obtained by simply requiring that $\pi \in\{0,1\}$ and we have [39]:

$$
\begin{array}{r}
\theta_{1}+\theta_{2}-\pi \leq 1 \\
-\theta_{1}-\theta_{2}+2 \pi \leq 0
\end{array}
$$

Now consider a binary variable $\theta$ and a non-negative real variable $r$. Assume that $r_{\max }$ is an upper bound for the real variable $r$. The quadratic term $r \theta$ can be replaced by a new non-negative real auxiliary variable $v$, such that its values correspond to the values of $r$ and $\theta$ as follows:

$$
v= \begin{cases}0 & \text { if } \theta=0 \\ r & \text { if } \theta=1\end{cases}
$$

The desired correspondence is obtained by simply requiring that $[40]$ :

$$
\begin{gathered}
0 \leq v \leq r \\
r-r_{\max }(1-\theta) \leq v \leq r_{\max } \theta
\end{gathered}
$$




\section{Proof of Lemma 1 and Lemma 2}

Proof of Lemma 1: Inequality (28) is obtained from (27) as follows.

$$
\begin{aligned}
\sum_{l, k:(l, k) \in V_{S}^{i}} \overline{\mathbf{x}}_{m n}^{T} \overline{\mathbf{x}}_{l k} f_{l k} & =\sum_{l, k:(l, k) \in V_{S}^{i} \backslash\left\{(l, k): \overline{\mathbf{x}}_{m n}^{T} \overline{\mathbf{x}}_{l k}=0\right\}} f_{l k} \\
& <\sum_{l, k:(l, k) \in V_{S}^{j} \backslash\left\{(l, k): \overline{\mathbf{x}}_{p q}^{T} \overline{\mathbf{x}}_{l k}=0\right\}} f_{l k} \\
& =\sum_{l, k:(l, k) \in V_{S}^{j}} \overline{\mathbf{x}}_{p q}^{T} \overline{\mathbf{x}}_{l k} f_{l k}
\end{aligned}
$$

From (11) and (42), we have:

$$
\sum_{l, k:(l, k) \in V_{S}^{i}} \overline{\mathbf{x}}_{m n}^{T} \overline{\mathbf{x}}_{l k} f_{l k}<\varepsilon
$$

Eq. (29) results from replacing (43) in (26).

Proof of Lemma 2: Consider a maximal clique $Q_{S}^{i} \in \mathbb{Q}_{S}$ so that $(m, n),(p, q) \in V_{S}^{i}$. Since $\overline{\mathbf{x}}_{m n}^{T} \overline{\mathbf{x}}_{p q}=1$, we have:

$$
\overline{\mathbf{x}}_{m n}^{T} \overline{\mathbf{x}}_{l k}=\overline{\mathbf{x}}_{p q}^{T} \overline{\mathbf{x}}_{l k}, \quad \forall l, k:(l, k) \in V_{S}^{i}
$$

Thus,

$$
\sum_{l, k:(l, k) \in V_{s}^{i}} \overline{\mathbf{x}}_{m n}^{T} \overline{\mathbf{x}}_{l k}=\sum_{l, k:(l, k) \in V_{s}^{i}} \overline{\mathbf{x}}_{p q}^{T} \overline{\mathbf{x}}_{l k}
$$

Knowing that $\rho_{m n}^{i}(0)=\rho_{p q}^{i}(0)$, equality (30) directly follows from (26) and (45).

\section{ACKNOWLEDGMENT}

This work was supported by the Natural Sciences and Engineering Research Council of Canada (NSERC).

\section{REFERENCES}

[1] I. Akyildiz and X. Wang, "A survey on wireless mesh networks," IEEE Communications Magazine, vol. 43, pp. 23-30, Sept. 2005.

[2] P. Bahl, A. Adya, J. Padhye, and A. Wolman, "Reconsidering wireless systems with multiple radios," ACM Computer Communication Review, vol. 34, pp. 39-46, Oct. 2004.

[3] A. Raniwala and T. Chiueh, "Architecture and algorithms for an IEEE 802.11-based multi-channel wireless mesh network," in Proc. of IEEE Infocom, Miami, FL, March 2005.

[4] P. Kyasanur and N. Vaidya, "Routing and interface assignment in multichannel multi-interface wireless networks," in Proc. of IEEE WCNC, New Orleans, LA, 2005.

[5] M. Marina and S. Das, "A topology control approach for utilizing multiple channels in multi-radio wireless mesh networks," in Proc. of Broadnet'05, Boston, MA, Oct. 2005.

[6] B. Raman, "Channel allocation in 802.11-based mesh networks," in Proc. of IEEE Infocom, Barcelona, Spain, April 2006.

[7] P. Kyasanur and N. Vaidya, "Capacity of multi-channel wireless networks: Impact of number of channels and interfaces," in Proc. of ACM MobiCom, Cologne, Germany, Sept. 2005.

[8] J. So and N. Vaidya, "Multi-channel MAC for ad hoc networks: Handling multi-channel hidden terminals using a single transceiver," in Proc. of ACM MobiHoc, Tokyo, Japan, May 2004.

[9] P. Bahl, R. Chandra, and J. Dunagan, "SSCH: slotted seeded channel hopping for capacity improvement in IEEE 802.11 ad hoc wireless networks," in Proc. of ACM MobiCom'04, Philadelphia, PA, Oct. 2004.

[10] A. Tzamaloukas and J. J. Garcia-Luna-Aceves, "A receiver-initiated collision-avoidance protocol for multi-channel networks," in Proc. of IEEE Infocom, Anchorage, Alaska, April 2001.

[11] M. Kodialam and T. Nandagopal, "Characterizing the capacity region in multi-radio multi-channel wireless mesh networks," in Proc. of ACM MobiCom, Cologne, Germany, Sept. 2005.

[12] A. Raniwala, K. Gopalan, and T. Chiueh, "Centralized channel assignment and routing algorithms for multi-channel wireless mesh networks," ACM Mobile Computing and Communications Review, vol. 8, pp. 50-65, April 2004.
[13] X. Meng, K. Tan, and Q. Zhang, "Joint routing and channel assignment in multi-radio wireless mesh networks," in Proc. of IEEE ICC, Istanbul, Turkey, June 2006.

[14] Y. Y. Chen, S. C. Liu, and C. Chen, "Channel assignment and routing for multi-channel wireless mesh networks using simulated annealing," in Proc. of IEEE Globecom, San Francisco, CA, November 2006.

[15] M. Alicherry, R. Bhatia, and L. E. Li, "Joint channel assignment and routing for throughput optimization in multi-radio wireless mesh networks," in Proc. of ACM MobiCom, Cologne, Germany, Sept. 2005.

[16] A. H. Mohsenian Rad and V. W. S. Wong, "Logical topology design and interface assignment for multi-channel wireless mesh networks," in Proc. of IEEE Globecom, San Francisco, CA, November 2006.

[17] J. Zhang, H. Wu, Q. Zhang, and B. Li, "Joint routing and scheduling in multi-radio multi-channel multi-hop wireless networks," in Proc. of Broadnet'05, Boston, MA, Oct 2005.

[18] J. Tang, G. Xue, and W. Zhang, "Interference-aware topology control and QoS routing in multi-channel wireless mesh networks," in Proc. of ACM MobiHoc, Urbana-Champaign, IL, May 2005.

[19] Y. Zhang and S. Qu, "Joint power control and channel assignment algorithms for wireless ad hoc networks," Lecture Notes in Computer Science, vol. 4159, pp. 270-279, August 2006.

[20] A. H. Mohsenian Rad and V. W. S. Wong, "Joint optimal channel assignment and congestion control in multi-radio wireless mesh networks," in Proc. of IEEE ICC, Istanbul, Turkey, June 2006.

[21] L. Chen, S. Low, and J. Doyle, "Joint congestion control and media access control design for ad hoc wireless networks," in Proc. of IEEE Infocom, Miami, FL, March 2005

[22] Z. Fang and B. Bensaou, "Fair bandwidth sharing algorithm based on game theory framework for wireless ad-hoc networks," in Proc. of IEEE Infocom, Hong Kong, China, March 2004.

[23] A. Bar-noy, A. Mayer, B. Schieber, and M. Sudan, "Guaranteeing fair service to persistent dependent tasks," SIAM Journal on Computing, vol. 27, pp. 1168-1189, Aug. 1998.

[24] K. Jain, J. Padhye, V. Padmanabhan, and L. Qiu, "Impact of interference on multi-hop wireless network performance," in Proc. of ACM MobiCom'03, San Diego, CA, Sept. 2003.

[25] J. Yellen and J. Gross, Graph Theory and Its Applications. Chapman \& Hall/CRC, 2005.

[26] G. Cornuejols, "The strong perfect graph conjecture," in Proc. of International Congress of Mathematics, Beijing, China, Aug. 2002.

[27] C. Shannon, "A theorem on coloring the lines of a network," J. Math. Phys., vol. 28, pp. 148-151, Sept. 1949.

[28] B. Hajeck and G. Sasaki, "Link scheduling in polynomial time," IEEE Trans. on Information Theory, vol. 34, pp. 910-917, Sept. 1988.

[29] E. Balas and C. Yu, "Finding a maximum clique in an arbitrary graph," SIAM Journal on Computing, vol. 15, pp. 1054-1068, Nov. 1986.

[30] J. Mo and J. Warland, "Fair end-to-end window-based congestion control," IEEE/ACM Trans. on Networking, vol. 8, pp. 556-567, Oct. 2000.

[31] R. Horn and C. Johnson, Topics in Matrix Analysis, 1st ed. Cambridge University Press, 1994.

[32] D. P. Bertsekas, Nonlinear Programming, 2nd ed. Athena Science, 2004.

[33] A. H. Land and A. G. Doig, "An automatic method for solving discrete programming problems," Econometrica, vol. 28, pp. 497-520, 1960.

[34] Z. Fang and B. Bensaou, "Design and implementation of a mac scheme for wireless mesh networks based on a cooperative game framework," in Proc. of ICC, Paris, France, June 2004.

[35] K. Kiwiel, "Convergence of approximate and incremental subgradient methods for convex optimization," SIAM Journal on Optimization, vol. 14, pp. 807-840, 2003.

[36] "ILOG CPLEX." http://www.ilog.com/products/cplex/, 2006.

[37] S. Low and D. Lapsley, "Optimization flow control, I: Basic algorithms and convergence," IEEE/ACM Trans. on Networking, vol. 7, pp. 861874, Dec. 1999.

[38] A. H. Mohsenian Rad and V. W. S. Wong, "Partially overlapped channel assignment for multi-channel wireless mesh networks," in Proc. of IEEE ICC (to appear), Glasgow, Scotland, June 2007.

[39] L. J. Watters, "Reduction of integer polynomial programming problems to zero-one linear programming problems," Operations Research, vol. 15, pp. 1171 - 1174, Nov. - Dec. 1967.

[40] F. Glover, "Improved linear integer programming formulations of nonlinear integer problems," Management Science, vol. 22, pp. 455 - 460, Dec. 1975. 